\title{
Multi-objective Parameter CPG Optimization for Gait Generation of a Quadruped Robot Considering Behavioral Diversity
}

\author{
Miguel Oliveira, Cristina P. Santos, Lino Costa, Vítor Matos and Manuel Ferreira
}

\begin{abstract}
This paper presents a gait multi-objective optimization system that combines bio-inspired Central Patterns Generators (CPGs) and a multi-objective evolutionary algorithm. CPGs are modeled as autonomous differential equations, that generate the necessary limb movement to perform the required walking gait. In order to optimize the walking gait, four conflicting objectives are considered, simultaneously: minimize the body vibration, maximize the velocity, maximize the wide stability margin and maximize the behavioral diversity. The results of NSGA-II for this multi-objective problem are discussed. The effect of the inclusion of a behavioral diversity objective in the system is also studied in terms of the walking gait achieved. The experimental results show the effectiveness of this multi-objective approach. The several walking gait solutions obtained correspond to different trade-off between the objectives.
\end{abstract}

\section{INTRODUCTION}

Legged robot locomotion applies nonlinear dynamical equations of high order with a multidimensional and irregular set of parameters. Therefore, hand-tuning is very hard to achieve and may not ensure the best results. Biological inspired Evolutionary Computation (EC) appears as the natural choice for gait optimization of legged robots. It is suitable for large dimension constrained multi-objective problems; robust in optimization problems where it is very difficulty to obtain local information and the objective functions are complex; and it is a model-free approach resistant to noise in the evaluation function. This is very convenient since it is very difficult to obtain the dynamical model of the legged robot. Furthermore, it provides for a strong global search capability with a minimum risk of getting stuck in a local minimum, and it may easily be deployed in parallel implementations in order to reduce the required computational time to find a solution.

Several gait optimization for biped, quadrupeds or hexapod robots have adopted EC models for optimization problems [2]. Robocup is one of the motivation engines for these works, specially using the AIBO quadruped robot.

Gait optimization using Genetic Algorithms (GAs) is the most used approach. In order to improve and adapt the GA to a specific gait optimization problem, it may be necessary to add some modifications. In [13], it is studied a crossover

\footnotetext{
Miguel Oliveira, Cristina P. Santos, and Vitor Matos are with Industrial Electronics Department, School of Engineering, University of Minho, 4800-058 Guimaraes, Portugal mcampos@dei.uminho.pt, cristina@dei.uminho.pt, vmatos@dei.uminho.pt, mjf@dei.uminho.pt

Lino Costa is with Production Systems Department, School of Engineering, University of Minho, 4710-057 Braga, Portugal lacedps.uminho.pt
}

operator with interpolation. Veloso [2] uses a two-point crossover, Gaussian mutation and overlapping populations to preserve the best individuals. Elitism strategies are also used in [16]. Adaptation strategies have been adopted in [2] to avoid premature convergence to local minima. Other Evolutionary Algorithms (EAs), such as Genetic Programming (GP) and Evolution Strategies (ESs) [9], have been used in the gait optimization problem.

In this study, we focus on the quadruped gait optimization. We propose an approach to optimize a specific quadruped gait, a slow crawl gait, which has to respect a large duty factor $\beta$. This is a very important slow statically stable gait with three legs in ground contact, suitable for postural control and therefore required to deal with movement in uneven terrain. In current works [2], [14], [9] the robot configuration is the one required to achieve higher velocities: the robot knees are completely folded and use a trot gait locomotion. Therefore, differently from current literature, in this work the aim is not to achieve the highest possible velocity in any gait, but rather to achieve the highest velocity for a slow crawl gait, which has to respect a given duty factor and certain phase relationships.

We take inspiration from the vertebrate biological motor systems [8] specificaaly in the concept of Central Pattern Generators (CPGs), pools of neurons that produce coordinated patterns of motor activity while being initiated and modulated by simple command signals. We base ourselves on previous work from others [12], [5] and from the team [16], [15], we apply (oscillator-based) differential equations to model a limb-CPG, that generates trajectories for hip robot joints [15]. These CPGs are modelled as coupled oscillators and solved using numerical integration. In order to achieve the desired crawl gait, it is necessary to appropriately tune the CPG parameters. The hand-tuning of these parameters is however difficult, and can only be obtained by trail and error or systematically. We combine the proposed CPG approach with an optimization strategy that searches for the best set of CPG parameters that result in the desired gait.

In this work speed, vibration and stability are the evaluated criteria used to explore the parameter space of the network of CPGs. The minimization of the body vibration [7], measured by accelerometers built-in the robot body, ensures a smooth locomotion (energy efficient) of the robot. By addressing both a speed and an energy related objective, it generally ensures that unrealistic behaviors (like high vibrations, jumps, etc.) are avoided.

We apply the elitist Nondominated Sorting Genetic Algo- 
rithm [4] (NSGA-II) that uses the dominance relation and a crowding mechanism to guide the search for the best CPG parameters, considering the described evaluation criteria. We used a hand-tuned gait as a seed to evolve the quadrupedal gait.The NSGA-II, was used for biped and quadruped [10]. In [11] a multi-objective optimization for gait generation was developed and applied MOEA. Slightly different objectives were considered. In the overall, the method was strongly model based in contrast to the work herein presented. Further, we have NSGA-II seems to have better performance [4].

Recently different approaches explore the idea of explicitly encourage diversity in the robot behavior space instead of in the space explored by the optimization algorithms. We follow the approach presented in [10], and define behavioral diversity in population as a objective to optimize. This assures the maximum diversity in the population. Herein we compute the distance between robot behaviors instead of the distance between genotypes [10].

Several experiments are performed in a simulated Aibo robot in order to assess the performance of the NSGAII on this multi-objective problem. The experimental results demonstrate that our approach allows to find solutions with lower vibration, higher velocity and higher wide stability margin for a quadruped slow crawl gait when compared to a previously hand-tuned gait. The quality of the latter was already considered good in absolute terms but could be improved with the NSGA-II. Further, we have observed that it was better to introduce behavioral diversity onto the multiobjective problem, since more diversified robot behaviors in terms of the legs movements were achieved. In the future, we plan to address other behavioral descriptors.

The problematic of evolving CPG parameters for robotic locomotion has started to be tackled a long time ago [1]. But, despite the fact that multi-objective algorithms had already been employed to tackle gait optimization [10] and the introduction of behavioral diversity onto the gait optimization problem had also been considered [10], the application of the chosen combination of technologies to quadruped walking is new (CPGs and NSGA-II), as well as the comparison of the conflict resolution mechanism and the number of evaluated objectives in the considered context of gait optimization.

\section{LOCOMOTION GENERATION}

\section{A. Gait Description}

In this work we address the crawl gait generation. This is a slow symmetric gait, meaning that the two legs of the same girdle are 0.5 out of phase. This gait is singular (two or more legs are simultaneously lifted or placed during a stride) and regular (all the legs have the same duty factor, $\beta$ ).

\section{B. Rhythmic Movement Generation}

Each hip joint of a limb, $i$, is directly controlled by the $x_{i}$ variable that evolves from a modified Landau-Stuart oscillator given by

$$
\begin{aligned}
\dot{x}_{i} & =\alpha\left(\mu-r_{i}^{2}\right)\left(x_{i}-O_{i}\right)-\omega z_{i}, \\
\dot{z}_{i} & =\alpha\left(\mu-r_{i}^{2}\right) z_{i}+\omega\left(x_{i}-O_{i}\right),
\end{aligned}
$$

where $r_{i}=\sqrt{\left(x_{i}-O_{i}\right)^{2}+z_{i}^{2}}$, amplitude of the oscillations is given by $A=\sqrt{\mu}, \omega$ specifies the oscillations frequency (in $\operatorname{rad} s^{-1}$ ) and relaxation to the limit cycle is given by $\frac{1}{2 \alpha \mu}$. The $z$ variable is an auxiliary variable required to assure periodic motion.

This oscillator contains an Hopf bifurcation from a stable fixed point at $\left(x_{i}, z_{i}\right)=\left(O_{i}, 0\right)$ (when $\mu<0$ ) to a structurally stable, harmonic limit cycle, for $\mu>0$.

The following expression for $\omega$ allows an independent control of speed of the ascending and descending phases of the rhythmic signal [12], meaning an independent control of the stance $\omega_{s t}$ and the swing durations $\omega_{\mathrm{sw}}$,

$$
\omega=\frac{\omega_{\mathrm{st}}}{1+e^{-a z_{i}}}+\frac{\omega_{\mathrm{sw}}}{1+e^{-a z_{i}}} .
$$

The alternation speed between these two values is controlled by $a$. The stance phase frequency, $\omega_{s t}$, is determined based on the constant swing frequency, $\omega_{\mathrm{sw}}$, and on the desired duty factor, $\beta$ as follows:

$$
\omega_{s t}=\frac{1-\beta}{\beta} \omega_{\mathrm{sw}}
$$

This set of equations models a CPG. It takes a set of parameters, $\mu \beta$, for the modulation of the generated trajectories for each hip joint.

All these parameters will be tuned by the optimization system described in section III, controlling the parameters for a locomotion that maximizes a fitness. The parameters $\alpha$, $\omega_{\mathrm{sw}}$ and $a$ are set a priori. Parameter $\omega_{\mathrm{sw}}$ specifies the swing phase duration, which is kept constant. Its value depends on the desired speed of movements and on the capabilities of the robotic platform.

\section{Interlimb Coordination}

We have four CPGs, one for each Hip joint. These four CPGs are coupled in order to achieve the limb coordination required in a walking gait pattern. The applied coupling scheme is depicted in Figure 1 and is given by

$$
\begin{array}{r}
{\left[\begin{array}{c}
\dot{x}_{i} \\
\dot{z}_{i}
\end{array}\right]=\left[\begin{array}{cc}
\alpha\left(\mu-r_{i}^{2}\right) & -\omega \\
\omega & \alpha\left(\mu-r_{i}^{2}\right)
\end{array}\right]\left[\begin{array}{c}
x_{i}-O_{i} \\
z_{i}
\end{array}\right]} \\
+\sum_{j \neq i} \mathbf{R}\left(\theta_{i}^{j}\right)\left[\begin{array}{c}
x_{j}-O_{j} \\
z_{j}
\end{array}\right] .
\end{array}
$$

The linear terms are rotated onto each other by the rotation matrix $\mathbf{R}\left(\theta_{i}^{j}\right)$, where $\theta_{i}^{j}$ is the required relative phase among the $i$ and $j$ hip oscillators to perform the gait (we exploit the fact that $\mathbf{R}(\theta)=\mathbf{R}^{-1}(-\theta)$ ).

The generated $x_{i}$ solution of this network of oscillator is used as the control trajectory for a Hip joint of the robot limbs $(i=\{\mathrm{LF}, \mathrm{RH}, \mathrm{RF}, \mathrm{LH}\})$. These trajectories encode the values of the joint's angles $\left(^{\circ}\right)$ and are sent online for the lower level PID controllers of each limb joint.

The knee joints are controlled as simple as possible. When the limb performs the swing (stance) phase, the knee flexes to a fixed angle, $K_{\mathrm{FL}},\left(K_{\mathrm{FL}, \min }\right)$. 


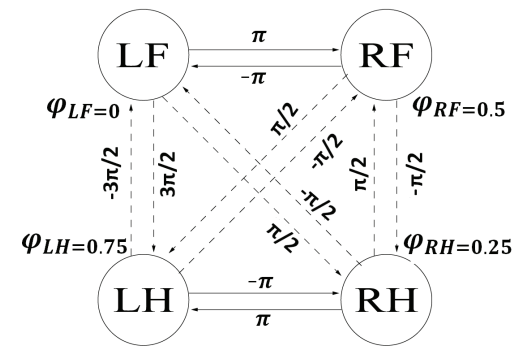

Fig. 1. Each leg lags one quarter of a cycle, in the sequence: Left Fore, Right Hind, Right Fore, Left Hind. In the arrows is indicated the relative phase among hip CPGs.

\section{OPTIMIZATION SYSTEM}

The proposed network of CPGs generates trajectories for the robot limbs. Different combinations of these trajectories for each joint in terms of amplitude, offset and frequency, result in different gait patterns. The proposed CPGs are based on Hopf oscillators. They have the intrinsic property of smoothly modulate the generated trajectories according to explicit changes in the CPG parameters: amplitude $\mu$, offset $O$ and the stance knee value. Therefore, we have to tune the CPG parameters.

We use an optimization algorithm framework based on NSGA-II to search for the optimal set of the CPG parameters. A scheme of the optimization system is depicted in Figure 2.

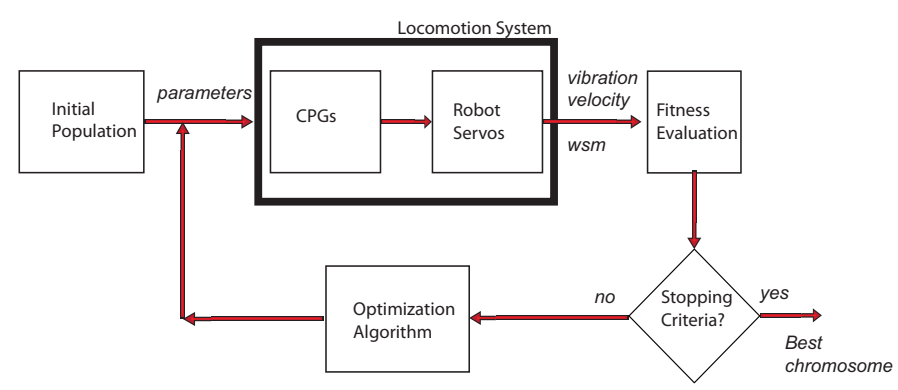

Fig. 2. Optimization Locomotion System

\section{A. Multi-objective formulation}

Mathematically, a multi-objective optimization problem with $s$ objectives and $n$ decision variables can be formulated as, without loss of generality:

$$
\begin{aligned}
& \min \mathbf{f}(\mathbf{x})=\left(f_{1}(\mathbf{x}), \ldots, f_{s}(\mathbf{x})\right) \\
& \text { subject to } \quad \mathbf{g}(\mathbf{x}) \geq 0 \text { and } \mathbf{h}(\mathbf{x})=0
\end{aligned}
$$

where $\mathbf{x} \in \mathbb{X} \subset \mathbb{R}^{n}$ is the decision vector defined in the decision space $\mathbb{X}=\left\{\mathbf{x} \in \mathbb{R}^{n}: \mathbf{l} \leq \mathbf{x} \leq \mathbf{u}\right\}, \mathbf{f}(\mathbf{x}) \subset \mathbb{F}^{s}$ is the objective vector defined in the objective space $\mathbb{F}^{s}, \mathbf{g}(\mathbf{x})$ and $\mathbf{h}(\mathbf{x})$ are the inequality and equality constraints, respectively.

Solving multi-objective problems is a very difficult task due to, in general, for this class of problems, the objectives conflict across a high-dimensional problem space and the computational complexity of the problem. Thus, the interaction between the multiple objectives gives rise to a set of efficient solutions, known as Pareto-optimal solutions.

For a multi-objective minimization problem, a solution a is said to dominate a solution $\mathbf{b}$, if and only if, $\forall i \in\{1, \ldots, s\}$ : $f_{i}(\mathbf{a}) \leq f_{i}(\mathbf{b})$ and $\exists j \in\{1, \ldots, s\}: f_{j}(\mathbf{a})<f_{j}(\mathbf{b})$. A solution $\mathbf{a}$ is said to be non-dominated regarding a set $\mathbb{Y}^{n} \subseteq \mathbb{X}^{n}$ if and only if, there is no solution in $\mathbb{Y}^{n}$ which dominates a. The solution $\mathbf{a}$ is Pareto-optimal if and only if $\mathbf{a}$ is non-dominated regarding $\mathbb{X}^{n}$.

The main goal of a multi-objective algorithm is to find a good and balanced approximation to the Pareto-optimal set. Note that Pareto-optimal parameter vectors cannot be improved in any objective without causing degradation in at least one of the other objectives. In this sense they represent globally optimal solutions.

\section{B. Specification of Objectives}

In this work, we consider four objectives to measure the performance of a walk gait: the vibration, the wide stability margin, the velocity and the behavioral diversity.

1) Vibration: We consider that a good gait should have less vibration, because the robot is subjected to less strain. In order to calculate the total vibration we sum the standard deviation of the measures of the $\left(a_{x}, a_{y}, a_{z}\right)$ accelerometers built-in onto the robot, similarly to [7], [13], [14], as follows:

$$
f_{a}=\operatorname{std}\left(a_{x}\right)+\operatorname{std}\left(a_{y}\right)+\operatorname{std}\left(a_{z}\right)
$$

2) Wide Stability Margin: For stability, we calculate the wide stability margin [17] (WSM). This is a measure of the locomotion stability that provides the shortest distance between the projection of the center of mass in the ground and the polygon formed by the vertical projection in the ground of robot feet contact points. A gait is considered better for higher WSM values.

3) Velocity: We calculate forward velocity using the traveled distance of the robot during the evaluation of each chromosome of the population, i.e. during 12 seconds. A gait is considered better if it achieves higher velocities.

4) Behavioral Diversity: The Behavioral diversity objective is to maximize the average distance of each chromosome to the other chromosomes of the population. We use the Hamming distance to calculate this behavioral distance by exclusively relying on sensory-motor values. Our implementation is similar to the one described in [10].

We consider discretized leg positions at each time step, described by a set of effector, $e$, and sensor, $s$, data as follows. The movement of each leg is divided into four instants during a stride, two during the stance phase and two during the swing phase, corresponding to the moments a leg is stretched forward or backwards. At each time step, each of these bits is set to 1 if the leg is in the corresponding position and 0 otherwise.

In the overall we define 16 effector values at time $t$, four for each Limb, $e=\left[\left\{e_{0}(t), ., e_{n}(t)\right\}, t \in[0, T]\right]$, where $n=15$ and $T$ is the observation length $(T=12 \mathrm{~s})$. 
TABLE I

PARAMETER BOUNDS

\begin{tabular}{|l|c|c|c|c|c|c|c|}
\hline & $\mu_{F L}$ & $O_{F L}$ & $\mu_{H L}$ & $O_{H L}$ & $\omega_{\mathrm{sw}}(\mathrm{rad} / \mathrm{s})$ & $K_{F L}\left({ }^{\circ}\right)$ & $K_{H L}\left({ }^{\circ}\right)$ \\
\hline Upper & 3600 & 400 & 3600 & 1600 & 12 & 127 & 127 \\
\hline Lower & 0.0001 & -1600 & 0.0001 & -400 & 1 & -30 & 5 \\
\hline
\end{tabular}

The sensor vector $s(t)$ is composed from perceptions at time $t$, i.e. the values coming from the considered sensors. Herein, we only consider the WSM measures as follows

$$
s(t)=\left\{\begin{array}{ll}
1 & \text { if } \operatorname{WSM}(t) \geq 0 \\
0 & \text { otherwise }
\end{array} .\right.
$$

This enables us to build up the behavior feature vector, $\vartheta(t)$, with 17 elements (16 effectors and 1 sensor values). For each time step we have $\vartheta(t)=\left[\left\{e_{0}(t), ., e_{n}(t), s(t)\right\}, t \in\right.$ $[0, T]]$, where $n=15$. The distance value is calculated using the Hamming distance that counts the number of bits that differ between two binary sequences. The binary sequences that describe the behavior of the robot at each time step correspond to the binary versions of $\vartheta$ and are computed as follows: $\vartheta_{\text {bin }}(t)=\left[\left\{e_{\operatorname{bin}_{0}}(t), \ldots, e_{\operatorname{bin}_{n}}(t), s(t)\right\}, t \in[0, T]\right]$, where $n=15$.

The total behavior distance of the chromosome is given by the sum of distances to each chromosome of the population:

$$
f_{\text {dist }}\left(\vartheta_{i}\right)= \begin{cases}\sum_{j=0}^{P} \sigma\left(\vartheta_{i}, \vartheta_{j}\right) & \forall i \neq j \\ 0 & \forall i=j\end{cases}
$$

where $\sigma\left(\vartheta_{i}, \vartheta_{j}\right)$ is the Hamming distance between two behavior feature vectors $\vartheta_{i}$ and $\vartheta_{j}$ in a time step.

\section{Fitness Specification}

Therefore, the mathematical formulation of this multiobjective problem involves four objectives:

$$
f=\left\{\begin{array}{l}
\min (f a) \\
\max (\text { vel }) \\
\max (W S M) \\
\max \left(f_{\text {dist }}\right)
\end{array}\right.
$$

It should be noted that several constraints have to be imposed in this problem, as described in the following section.

\section{Constraint handling}

The search range of the CPG network parameters depend on the Aibo Ers-7 robot and as such are set before hand as shown in Table I.

Maximum and minimum values for each knee angle are calculated in order to avoid leg collision during locomotion. Therefore, inequality constraints have been introduced to prevent invalid solutions.
In order to handle the simple boundary constraints, each new generated point is projected component by component in order to satisfy boundary constraints as follows:

$$
x_{i}= \begin{cases}l_{i} & \text { if } x_{i}<l_{i} \\ x_{i} & \text { if } l_{i} \leq x_{i} \leq u_{i} \\ u_{i} & \text { if } x_{i}>u_{i}\end{cases}
$$

where $l_{i}, u_{i}$ are the lower and upper limit of $i$ component, respectively. In order to handle inequality constraints, the tournament based constraint method proposed by Deb [3] that is based on a penalty function which does not require any penalty parameter is adopted. For comparison purposes, tournament selection is exploited to make sure that:

1) when two feasible solutions are compared, the one with better objective function value is chosen;

2) when one feasible and one infeasible solutions are compared, the feasible solution is chosen;

3) when two infeasible solutions are compared the one with smaller constraint violation is chosen.

\section{E. NSGA-II algorithm}

We used the NSGA-II [4] that is a population based algorithm implementing techniques inspired by evolutionary biology such as inheritance, mutation, selection, and crossover.A population of points that represent potential Pareto optimal solutions to the problem being solved are, usually, referred to as chromosomes. NSGA-II uses ranking and crowding distance for fitness definition (see eq.9) and calculation [4]. Each generation, solutions in the population that are not dominated by any other solution solutions will constitute the 1 st front. Thereafter, the solutions of the $1 \mathrm{st}$ front are ignored temporarily, and the remaining solutions are processed. To the second level of non-dominated solutions is assigned a fitness value superior to the worst computed fitness value from the solutions in the 1st front. Solutions are also differentiated by the crowding distance which indicates the extent that the objective space area in which the solution resides is crowded by other solutions.

Tournament selection is used for mating selection in NSGA-II which among two randomly chosen solutions, the one that dominates the other is selected. If no one dominates the other, it is selected the one in the less crowded area. Thus, NSGA-II selects the solutions with the higher rank as first priority followed by the crowding distance to select from the same rank.

The search results from the creation of new chromosomes from old ones by the application of genetic operators. The crossover operator takes two randomly selected chromosomes; one point along their common length is randomly selected, and the characters of the two parent strings are swapped, thus generating two new chromosomes. The mutation operator, randomly selects a position in the chromosome and, with a given probability, changes the corresponding value. This operator introduces diversity in the population since selection and crossover, exclusively, could not assure the exploration of new regions in the search space. In order to 
recombine and mutate chromosomes, the Simulated Binary Crossover (SBX) and Polynomial Mutation were considered, respectively. These operators simulate the working of the traditional binary operators.

\section{EXPERIMENTAL RESULTS}

In order to address the desired goals and to verify the proposed solutions, several experiments were conducted:

1) NSGA-II(3OBJ): optimization system with the NSGA-II to optimize, simultaneously, three objectives: vibration $\left(f_{a}\right)$, velocity (vel) and wide stability margin $($ WSM);

2) NSGA-II(4OBJ):optimization system with the NSGAII to optimize, simultaneously, four objectives: vibration $\left(f_{a}\right)$, velocity ( $\left.v e l\right)$, wide stability margin (WSM) and behavior distance $\left(f_{\text {dist }}\right)$.

Since we are interested in the evaluation of the impact of the behavioral diversity on the obtained solutions, we have also calculated the behavioral distance objective $\left(f_{\text {dist }}\right)$ for NSGA-II(3OBJ).

In this work, real representation of the variables was considered. So, each chromosome consists of a vector of 7 real values representing the decision variables of the problem. Further, we used a hand-tuned gait as a seed to evolve the quadrupedal gait. In all experiments, we consider a population size of 100 chromosomes and a maximum number of generations of 50. The SBX crossover and polynomial mutation probabilities were, respectively, 0.9 and $1 / 7$.

For chromosome evaluation purpose, we consider the ers-7 AIBO dog robot that is a 18 DOFs quadruped robot made by Sony. Simulations were carried out in Webots, a simulation software based on ODE, an open source physics engine for simulating 3D rigid body dynamics. The locomotion controller generates trajectories for the hip and knee joint angles, that is 8 DOFs of the robot, 2 DOFs in each leg. At each sensorial cycle (30 ms), sensory information is acquired. We apply the Euler method with $1 \mathrm{~ms}$ fixed integration step, to integrate the system of equations.

Each chromosome is evaluated during 12 seconds. At the end of each chromosome evaluation the robot is set to its initial position and rotation, such that initial conditions are equal for the evaluation of all chromosomes of all populations. Results were obtained in an AMD Athlon XP 2400+2.00 Ghz (512 MB of RAM) PC.

Since we are solving a multi-objective optimization problem, we have used the hypervolume metric [6] to assess the quality of the approximate Pareto-optimal set generated by each algorithm. All distances and volumes are measured in the objective space. The reference point has been selected by taking the approximation to the Nadir point based on the non-dominated solutions present in the final populations: $r=\{1.47,0.5827,-18.1,103408\}$.

In Table II, we introduce the behavioral distance value $\left(f_{\text {dist }}\right)$ in the computation of the hypervolume of the final population obtained in each experiment. The best hypervolume was obtained by the NSGA-II with behavioral diversity (NSGA-II(4OBJ) experiment). This finding highlights the relevance of including this objective in order to achieve solutions that represent different behavior of the robot gait.

In Table II, the mean, standard deviation and best value of behavioral diversity of final population is presented for the different experiments conducted. As expected, it can be seen that the highest average value for behavior diversity was obtained in the NSGA-II(4OBJ) experiments, which indicates the importance of the inclusion of the behavioral diversity objective in the optimization system.

TABLE II

BEHAVIOR DIVERSITY FOR THE TWO EXPERIMENTS

\begin{tabular}{|c|c|c|c|c|}
\hline Experiment & mean & std & best & Hypervolume \\
\hline NSGA-II(3OBJ) & $1.3618 e+005$ & $2.8802 e+004$ & $2.5043 e+005$ & $9.3037 e+008$ \\
\hline NSGA-II(4OBJ) & $1.4034 e+005$ & $2.1993 e+004$ & $2.2937 e+005$ & $9.6620 e+008$ \\
\hline
\end{tabular}

We have used the non-parametric Mann-Whitney statistical test to compute the probability of two different samples have the same mean value. This way we use the behavioral diversity to calculate the similarity of the different experiment. The p-value of the Mann-Whitney test between NSGA-II(3OBJ) and NSGA-II(4OBJ) experiments is 0.0682. This indicates that, in these two experiments, the mean behavioral diversity measures of the two sets of solutions achieved in each experiment can be considered similar. However, it should be stressed that this comparison reflects the central tendency of the behavioral diversity and does not take into account the relations with the other objectives. As previously mentioned, the hypervolume measure indicates the importance of considering the behavioral diversity objective in the optimization procedure.

The inclusion of the behavioral diversity objective largely increased the number of nondominated solutions found since the objective space dimension is also increased. Solutions having different behavioral diversity distances correspond to similar trade-off between the other objectives. In summary, the introduction of behavioral diversity enable us to achieve a Pareto-optimal set that has diversified robot behaviors in terms of the legs movements.

Further, we can observe a clear and expected trade-off between the different objectives that indicate the conflictive relationship among them (speed and vibration). Two of the most interesting features of the resulting Pareto front are the almost exponential relations between the velocity and the WSM and the velocity and the vibration, though it is more notorious in the former. When moving at high speeds the vibration has high values and the stability has lower values. The solutions have to be chosen depending on the final endeavor and some compromises have to be accepted.

The resultant behavior and the optimal gaits for 3 different results of Pareto front solutions, A, B and C, are shown in the attached video.Firstly, we choose a solution that considers maximum velocity and low WSM (point indicated with an A). This point corresponds to a medium value of vibration. The optimal gait generated by the chosen second 
TABLE III

PARETO FRONT SOLUTIONS

\begin{tabular}{|c|c|c|c|c|}
\hline \multirow{2}{*}{ Solution } & \multirow{2}{*}{ Experiment } & \multicolumn{3}{|c|}{ Objective functions } \\
\cline { 3 - 5 } & & vibration & velocity $(\mathrm{m} / \mathrm{s})$ & WSM \\
\hline \multirow{3}{*}{ A } & NSGA-II(3OBJ) & 0.121 & 0.117 & 4.09 \\
& NSGA-II(4OBJ) & 0.120 & 0.112 & 0.14 \\
\hline \multirow{2}{*}{ B } & NSGA-II(3OBJ) & 0.032 & 0.034 & 24.93 \\
\hline \multirow{2}{*}{ C } & NSGA-II(4OBJ) & 0.059 & 0.086 & 7.71 \\
\hline & NSGA-II(3OBJ) & 0.088 & 0.015 & 32.61 \\
NSGA-II(4OBJ) & 0.089 & 0.004 & 32.23 \\
\hline
\end{tabular}

solution (B) satisfies every objective functions. The vibration is decreased by $50.83 \%$ but on the other hand the value of the velocity and the WSM cost functions are decreased (increased) by $23.23 \%$ and $98,21 \%$, respectively. Finally, the third chosen solution (C) shows a very small value for velocity (decreased by $94.7 \%$ ) with a corresponding large value for the WSM and vibration, that increased by $76.08 \%$ and $32.76 \%$, respectively, relatively to solution B. Table III shows the values of each solution for the two experiments.

Comparing the solution B of the NSGA-II(4OBJ) experiment with the hand-tuned gait, the walking speed was improved by around (40.77\%), the wide stability by $36,57 \%$ and vibration of $25.44 \%$. The attached video shows the robot running the evolved controller and provides for a sense of how competent the improvement was.

\section{CONCLUSIONS AND FUTURE WORKS}

In this article, we have addressed the locomotion optimization of a quadruped robot that walks with a slow walking gait. A locomotion controller based on dynamical systems to model CPGs, generates quadruped locomotion. These CPG parameters are tuned by an optimization system. This optimization system combines CPGs and a multi-objective optimization method, NSGA-II.

Experiments were performed in the Webots robotics simulator. The aim was to compare the performance of the optimization method according to the several objective functions criteria. Further, we study the impact of the Behavior Diversity objective in the performance of the NSGA-II method. The best results were obtained by the NSGA-II experiment that used the behavior diversity as an objective function. However, both techniques were able to obtain a set of parameters adequate for the implementation of a quadruped walking gait with lower vibration, higher stability margin and higher velocity comparatively to an already efficient (in absolute terms) hand-tuned gait. Currently, we are using other optimization methods such as Particle Swarm Optimization (PSO) and Ant Colony Optimization (ACO). We will extend this optimization work to address other locomotion related problems, such as: the generation and switch among different gaits according to the sensorial information and the control of locomotion direction.

\section{ACKNOWLEDGMENTS}

This work is funded by FEDER Funding supported by the Operational Program Competitive Factors - COMPETE and National Funding supported by the FCT - Portuguese Science Foundation through project PTDC/EEACRO/ 100655/2008. Thanks to Dr. Stéphane Doncieux from the Institut des Systmes Intelligents et de Robotique (ISIR) of the Pierre and Marie Curie University (UPMC).

\section{REFERENCES}

[1] R. D. Beer and J. C. Gallagher. Evolving dynamical neural networks for adaptive behavior. Adaptive Behavior, 1(1):91-122, 1992.

[2] S. Chernova and M. Veloso. An evolutionary approach to gait learning for four-legged robots. In Proceedings 2004 IEEE/RSJ International Conference on Intelligent Robots and Systems, IROS 2004., volume 3 , pages 2562 - 2567 vol.3, sept.-2 oct. 2004

[3] K. Deb. An efficient constraint handling method for genetic algorithms. In Computer Methods in Applied Mechanics and Engineering, pages 311-338, 1998.

[4] K. Deb, A. Pratap, S. Agarwal, and T. Meyarivan. A fast and elitist multiobjective genetic algorithm: Nsga-ii. Evolutionary Computation, IEEE Transactions on, 6(2):182 -197, Apr. 2002.

[5] S. Degallier, L. Righetti, L. Natale, F. Nori, G. Metta, and A. Ijspeert. A modular bio-inspired architecture for movement generation for the infant-like robot icub. In 2nd IEEE RAS EMBS International Conference on Biomedical Robotics and Biomechatronics, (BioRob 2008)., pages $795-800$, oct. 2008.

[6] C. Fonseca, L. Paquete, and M. Lopez-Ibanez. An improved dimension-sweep algorithm for the hypervolume indicator. In Evolutionary Computation, 2006. CEC 2006. IEEE Congress on, pages 1157 - 1163, 2006.

[7] D. Golubovic and H. Hu. Evolving locomotion gaits for quadruped walking robots. Industrial Robot: An International Journal, 32:259 267, 2005.

[8] S. Grillner. Neurobiological bases of rhythmic motor acts in vertebrates. Science, 228(4696):143-149, 1985.

[9] M. Hebbel, W. Nistico, and D. Fisseler. Learning in a high dimensional space: Fast omnidirectional quadrupedal locomotion. In G. Lakemeyer, E. Sklar, D. Sorrenti, and T. Takahashi, editors, RoboCup 2006: Robot Soccer World Cup X, volume 4434 of Lecture Notes in Computer Science, pages 314-321. Springer Berlin / Heidelberg, 2007.

[10] S. Koos, J.-B. Mouret, and S. Doncieux. Crossing the reality gap in evolutionary robotics by promoting transferable controllers. In Proceedings of the 12th annual conference on Genetic and evolutionary computation, GECCO '10, pages 119-126, New York, NY, USA, 2010. ACM.

[11] J.-Y. Lee, M.-S. Kim, and J.-J. Lee. Multi-objective walking trajectories generation for a biped robot. In IEEE/RSJ International Conference onIntelligent Robots and Systems, 2004, IROS 2004. volume 4, pages 3853 - 3858 vol.4, sept.-2 oct. 2004.

[12] L. Righetti and A. Ijspeert. Pattern generators with sensory feedback for the control of quadruped locomotion. In IEEE International Conference on Robotics and Automation, (ICRA 2008), pages 819 -824 , may 2008

[13] T. Röfer. Evolutionary gait-optimization using a fitness function based on proprioception. In RobuCup, pages 310-322, 2004.

[14] M. Saggar, T. D'Silva, N. Kohl, and P. Stone. Autonomous learning of stable quadruped locomotion. In RoboCup-2006: Robot Soccer World Cup X, volume 4434 of Lecture Notes in Artificial Intelligence, pages 98-109. Springer Verlag, Berlin, 2007.

[15] C. P. Santos and V. Matos. Gait transition and modulation in a quadruped robot: A brainstem-like modulation approach. Robotics and Autonomous Systems, 59(9):620 - 634, 2011.

[16] C. P. Santos, M. Oliveira, A. M. A. C. Rocha, and L. Costa. Head motion stabilization during quadruped robot locomotion: Combining dynamical systems and a genetic algorithm. In IEEE International Conference on Robotics and Automation, (ICRA 2009)., pages 2294 -2299 , may 2009.

[17] S. Song and K. Waldron. Machines that Walk: The Adaptive Suspension Vehicle. MIT Press, Cambridge, 1989. 